\title{
Physical Properties Investigation of Reduced Graphene Oxide Thin Films Prepared by Material Inkjet Printing
}

\author{
Veronika Schmiedova, ${ }^{1}$ Jan Pospisil, ${ }^{1}$ Alexander Kovalenko, ${ }^{1}$ Petr Ashcheulov, \\ Ladislav Fekete, ${ }^{2}$ Tomas Cubon, ${ }^{1}$ Peter Kotrusz, ${ }^{3}$ Oldrich Zmeskal, ${ }^{1}$ and Martin Weiter ${ }^{1}$ \\ ${ }^{1}$ Faculty of Chemistry, Brno University of Technology, Purkynova 118, 61200 Brno, Czech Republic \\ ${ }^{2}$ Institute of Physics, Academy of Sciences Czech Republic v. v. i., Na Slovance 2, 18221 Prague 8, Czech Republic \\ ${ }^{3}$ Danubia NanoTech, s.r.o., Ilkovicova 3, 84104 Bratislava, Slovakia \\ Correspondence should be addressed to Veronika Schmiedova; xcschmiedova@fch.vut.cz
}

Received 23 March 2017; Revised 12 July 2017; Accepted 19 July 2017; Published 23 August 2017

Academic Editor: Zainovia Lockman

Copyright (C) 2017 Veronika Schmiedova et al. This is an open access article distributed under the Creative Commons Attribution License, which permits unrestricted use, distribution, and reproduction in any medium, provided the original work is properly cited.

\begin{abstract}
The article is focused on the study of the optical properties of inkjet-printed graphene oxide (GO) layers by spectroscopic ellipsometry. Due to its unique optical and electrical properties, GO can be used as, for example, a transparent and flexible electrode material in organic and printed electronics. Spectroscopic ellipsometry was used to characterize the optical response of the GO layer and its reduced form (rGO, obtainable, for example, by reduction of prepared layers by either annealing, UV radiation, or chemical reduction) in the visible range. The thicknesses of the layers were determined by a mechanical profilometer and used as an input parameter for optical modeling. Ellipsometric spectra were analyzed according to the dispersion model and the influence of the reduction of GO on optical constants is discussed. Thus, detailed analysis of the ellipsometric data provides a unique tool for qualitative and also quantitative description of the optical properties of GO thin films for electronic applications.
\end{abstract}

\section{Introduction}

Graphene is a two-dimensional (2D) carbon-based material [1], which has recently attracted particular attention due to its specific electronic structure [2], which gives it unusual electronic properties such as the anomalous quantum Hall effect $[3,4]$ and startling high carrier mobility at relatively high charge carrier concentrations [5]. This material was first isolated in 2003 [6] and immediately became a potential candidate for electronic applications [7]. Recently, it was shown [8] that graphene also provides extraordinary optical properties. Graphene absorbs a $2.3 \%$ fraction of incident white light, and due to this graphene provides unique electronic properties and structure $[9,10]$. However, graphene sheets have limited practical application due to the fact that graphene is insoluble and infusible. Recent reports suggest that solution-processable graphene oxides (GOs), resulting from exfoliation of graphite powders with strong oxidizing reagents $[11,12]$, can be deposited by wet processing with a consequent reduction to a so-called reduced graphene oxide (rGO).

Graphene oxide (GO) is an atomically thin carbon nanostructure with various oxygen-containing functional moieties such as carbonyl, carboxyl, and hydroxyl (Figure 1). GO and rGO have attracted a great deal of attention due to their remarkable physical, chemical, and mechanical properties and could be used as a potential novel electronic material [1315].

Graphene oxide is potentially usable due to its simple synthesis and processing [12]. The presence of oxygen functional groups leads to dispersibility in water and other organic solvents [16]. This remains a very important feature for improving their properties of the material with polymer matrixes such as electrical and mechanical characterization. From previous studies in the literature [17], it is known that in graphene one absorbed photon is capable of producing many excited electrons (in traditional materials, energy from one photon only excites one electron) and is therefore able 


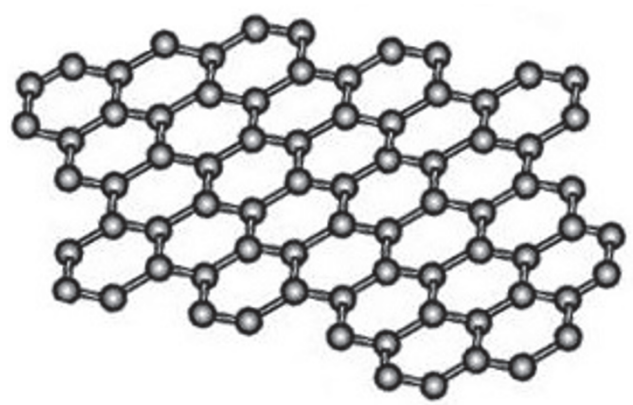

(a)

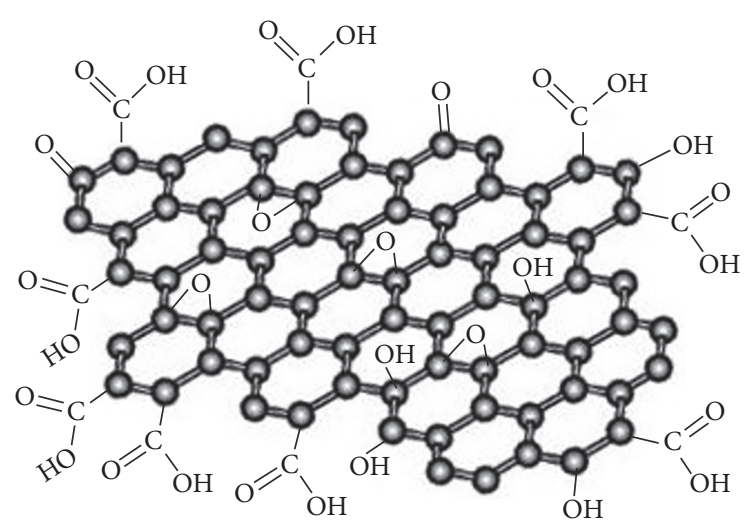

(b)

FIGURE 1: (a) Graphene structure and (b) GO structure with omission of minor groups (carboxylic, carbonyl, etc.).

to drive a greater photocurrent. Due to the very low level of light absorption [9] and concurrently high electron mobility of graphene, this material can be used as an alternative to ITO in the interlayer of photovoltaic cells [18].

GO is often described as an electrical insulator due to the disruption of its $\mathrm{sp}^{2}$ bonding networks. The relationship between the structure and properties of GO and rGO films can be assessed by the characterization of its oxidation degree. This can be done by the evaluation of the $\mathrm{sp}^{2}$ carbon fraction, since the optical and electrical properties are mostly governed by the $\pi$-electrons from $\mathrm{sp}^{2}$ carbon atoms $[19,20]$.

Several techniques for GO reduction have been reported, including thermal annealing in vacuum [21] or inert atmosphere [22], electrical reduction [23], chemical reduction by hydrazine [21, 24] or acids [25], and reduction by UV light [26]. Interestingly, with the degree of reduction, the work function of graphene layers can be fine-tuned from 5.2 to $4.5 \mathrm{eV}$ [27]; thus graphene oxide can be used as a holetransporting selective electrode; inasmuch as superior p-type behavior with hole mobilities up to $0.59 \mathrm{~cm}^{2} \mathrm{~V}^{-1} \mathrm{~s}^{-1}$ in thinfilm transistors has been reported [28].

One of the possible applications of rGO-based transparent conductors is as electrodes for photovoltaic devices (ITO replacement) [29]. An important criterion for replacing ITO in such devices is the work function of the material, which is higher than that of ITO, which means a higher hole selectivity of the contact. The work function of graphene is $4.5 \mathrm{eV}$, whereas that of ITO is $4.0-4.5 \mathrm{eV}$ [30].

The main goal of this paper is a thorough study of inkjetprinted [31] rGO layers' optical properties depending on the reduction method. Generally, inkjet technique is one of the most prospective ways to prepare cost-effective large-area deposition techniques for devices on a large variety of substrates regardless of their shape and thermal stability. Electrically functional electronic or optical inks including conducting or semiconducting materials of organic, inorganic, or hybrid origin can be deposited on the substrate, creating active or passive devices such as thin film transistors, diodes capacitors, coils, and resistors.
Inkjet printing technique of electronic devices offers a way of producing thin, lightweight, flexible, and environmentally friendly products, which can be extremely cost-effective compared to inorganic devices prepared by traditional techniques. Additionally, printable electrical components can be integrated into devices fabricated by a conventional way. Organic light-emitting diode (OLED) displays, smart clothing, solar cells, flexible displays, printed batteries, supercapacitors, memory, or label-protection tags are just a few examples of the promising application of systems based on printed electronics.

\section{Materials and Methods}

2.1. Material Characterization and Preparation. Graphene oxide flakes, supplied by Danubia NanoTech, were prepared by chemical oxidation (Han et al. modified Hummer's method [32]) of well-crystalline graphite powder. These nanosheets of graphite oxide are hydrophilic, so they can be easily dispersed in water. The concentration of GO dispersion was $4 \mathrm{mg} / \mathrm{ml}$.

Silica substrates with dimensions of $(1 \times 1) \mathrm{cm}$ were used for the deposition of GO layers. The substrates were sonicated in a bath containing diluted detergent Neodisher ${ }^{\circledR}$ (Miele) in deionized water (1:20 ratio). Subsequent rinsing in deionized water, isopropyl alcohol (p.a.), and chloroform (p.a.) was conducted just before deposition.

The thin GO layer deposition and patterning were carried out using an inkjet printer Fujifilm Dimatix 2831. The drop formation characteristics were observed via built-in stroboscopic camera. Interactions between printed layers and substrate were studied via optical microscope. Stable inkjet printing conditions were secured by stirring the GO solution for 1 hour at room temperature prior to filling it into the printing cartridge. Optimal printing conditions were defined as follows: 8 nozzles, $20 \mathrm{~V}$ driving voltage, nozzle temperature $30^{\circ} \mathrm{C}$, substrate temperature $45^{\circ} \mathrm{C}$, and the nozzle span was kept constant at $20 \mu \mathrm{m}$ (at an angle of $4.5^{\circ}$ ). A stock solution ideally contained $2 \mathrm{ml}$ of graphene, $0.8 \mathrm{ml}$ of ethanol, $0.8 \mathrm{ml}$ 
of isopropyl alcohol, and $0.2 \mathrm{ml}$ of hexanol. This solution was filtered through a $0.45 \mu \mathrm{m}$ cellulose filter, stirred for 60 minutes, and then immediately printed.

Chemically reduced GO films were prepared through incubation of the GO film in vapors of hydrazine [21, 24, 30]. GO films on quartz slides were placed into a glass Petri dish inside a larger glass Petri dish which also contained $1 \mathrm{~mL}$ of hydrazine. The larger dish was covered with a glass lid, sealed with Parafilm tape, and left at room temperature in a laboratory fume hood for $30 \mathrm{~min}$. Concerning the thermal reduction process, the samples were mounted onto a hot plate and heated to $200^{\circ} \mathrm{C}$ for $4 \mathrm{~min}$. Another possibility for GO reduction is UV reduction (rUV): samples were treated by $\mathrm{UV}$ irradiation $\left(3 \mathrm{~mW} / \mathrm{cm}^{2}\right)$ for $60 \mathrm{~min}$.

\subsection{Characterization of Methods. A UVISEL 2 Horiba Jobin} Yvon spectroscopic ellipsometer was used for measuring the wavelength dependence of the principal ellipsometric parameters $\Psi$ and $\Delta$ at an angle of $70^{\circ}$. Data obtained from ellipsometric measurement was evaluated using the commercial DeltaPsi2 program. The dispersion model used was the so-called "New Amorphous" model, which was derived by Horiba Jobin Yvon on the basis of Forouhi-Bloomer formulation [33-35].

Acquisition of the absorption spectra of the thin films was carried out by Varian Cary $50 \mathrm{UV}$-vis spectrophotometer (Agilent Technologies). Baseline correction was determined by measurement of the absorption spectra of a plain quartz glass. Subsequently the Cary WinUV software was used to process the obtained spectra.

A Dektak XT (Bruker Corporation) profilometer was used to evaluate the physical thickness of the deposited films. A stylus diameter of $12.5 \mu \mathrm{m}$ and applied force of $5 \mathrm{mg}$ were set to ensure reliable data acquisition. Vision64 software was employed to record and assess profilometric data.

Atomic force microscopy (AFM) using a Dimension Icon (Bruker) in Peak Force Tapping Mode with Tap150AL-g tips optimized for surface topography and surface roughness was measured on a $5 \times 5 \mu \mathrm{m}^{2}$ surface area.

Raman spectra were recorded by Renishaw InVia Raman spectrometer; spectra were corrected using the glass substrate as a baseline. Laser excitation wavelength of $488 \mathrm{~nm}$ with laser power at sample of $6 \mathrm{~mW}, 50 \mathrm{x}$ Olympus objective, $65 \mu \mathrm{m}$ slits, spot focus, and grating of 2400 lines $/ \mathrm{mm}$ were set to measurement. The spectrometer was calibrated by the F1g mode of silicon at $520.2 \mathrm{~cm}^{-1}$.

After the reduction process, the sheet resistance to the four-point probe method was determined using a Keithley 2100 analyzer (Tektronix Company).

\section{Results and Discussion}

The surfaces of the rGO inkjet-printed layers were investigated using atomic force microscopy (AFM). As shown in Figure 2, the roughness of the printed GO thin films is about 2-3 $\mathrm{nm}$, which proves lateral distribution of the individual graphene oxide flakes on a substrate. No holes or cracks were observed. Thus, it can be assuredly assumed that inkjet printing is an appropriate technique for wet processing of $\mathrm{GO}$ flakes' aqueous dispersion.

Figure 3 shows the absorption spectra of prepared samples of GO and its reduced form. For illustrative purposes a zoomed peak (200-400 nm) is depicted in the figure inset. It is very important to note that all reduction conditions were specified so as to remove the peak at about $300 \mathrm{~nm}$ (see inset Figure 3) [36], because this is a necessary step from the viewpoint of the electrical properties (carbon bonds with $\mathrm{sp}^{3}$ hybridization are removed) as was reported in $[37,38]$.

Highly symmetrical covalent carbon-carbon bonds of carbon-based materials are naturally having little or no natural dipole moment. Raman spectroscopy, which is one of the convenient techniques to investigate carbon-based materials [39], is the most sensitive technique to such type of bonds as far as the carbon-carbon bonds fit this criterion perfectly and as a result Raman spectroscopy is highly sensitive to these materials and capable of discerning even slight changes in structure making it a very valuable tool in the characterization of GO and rGO samples.

In the present case, the spectrum confirmed the presence of graphene oxide and the influence of its reduction (see Figure 4). The spectra of graphene have two significant Raman signals of weak intensity, $\mathrm{G}$ and $\mathrm{D}$ bands appearing at about $1575 \mathrm{~cm}^{-1}$ and $1350 \mathrm{~cm}^{-1}$, respectively, and a second order peak $2 \mathrm{D}$ at $2700 \mathrm{~cm}^{-1}$ which represents double resonance of scattered photons by excitation wavelength [40]. The $\mathrm{G}$ band corresponds to the pure natural graphite (i.e., peak of graphite) and is due to the vibration of carbon atoms with $\mathrm{sp}^{2}$ hybridization. The D band is a so-called peak of defects and is caused by vibration of carbon atoms with $\mathrm{sp}^{3}$ hybridization (i.e., a failure of graphene structure). These peaks of graphene oxide are slightly shifted and are now located at approximately $1592 \mathrm{~cm}^{-1}$ and $1346 \mathrm{~cm}^{-1}$, respectively (see Figure 4). This shift is due to destruction of $\mathrm{sp}^{2}$ bonds and the formation of defects on graphene sheets caused by their extensive oxidation. The ratio of the intensities of the $D$ and $G$ peaks $\left(I_{\mathrm{D}} / I_{\mathrm{G}}\right)$ allows comparing the density of defects in the samples: the higher the value is, the more the defects appear in the graphene structure. The value of the ratio $I_{\mathrm{D}} / I_{\mathrm{G}}$ in the case of thermal reduction is 1.079 , while in the case of chemical reduction it is 1.104 and for the UV reduction it is 0.821 . From these values, it follows that the most defects are found in chemically reduced graphene oxide. This is due to a higher amount of unreduced functional groups on graphene oxide. The reduction also increases the intensity of the value $2 \mathrm{D}$ peak; in the case for thermal reduction it is 0.27 and for the chemical reduction it is 0.21 , and in the case of the UV reduction it is 0.13 . From this, it follows that the UV reduction of graphene oxide is the least reduced.

In order to determine the optical constants of prepared layers (i.e., refractive index, extinction coefficient, and thickness layers), ellipsometric measurements have to be made. The optical properties of materials are significant primarily for evaluating experiments focused on the study of electronic processes. Examples of the processed spectra which depict the experimental and calculated ellipsometric data expressed 


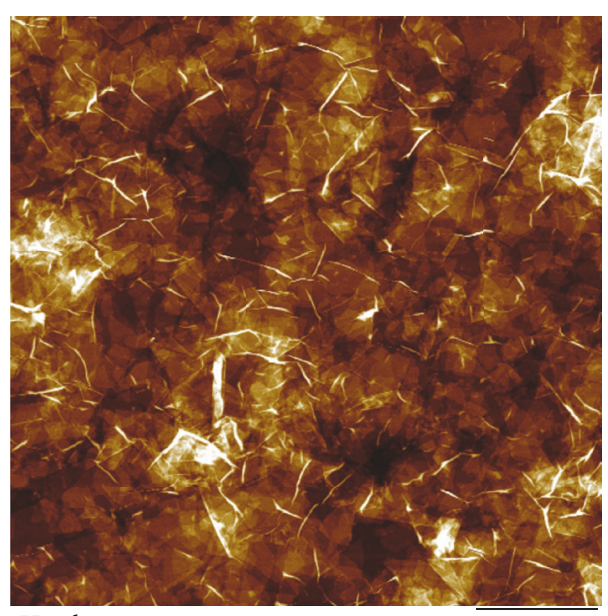

Height sensor

$1.0 \mu \mathrm{m}$

(a)

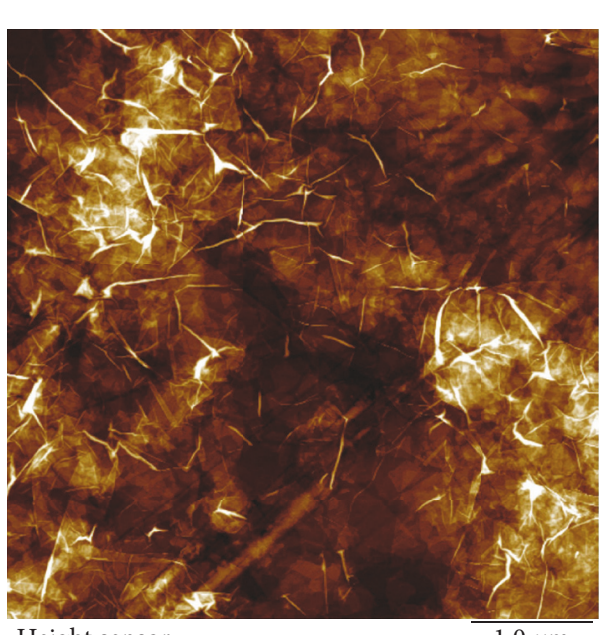

Height sensor

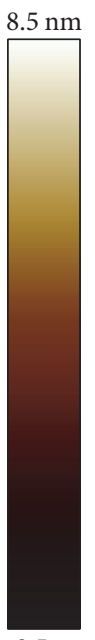

$-8.5 \mathrm{~nm}$

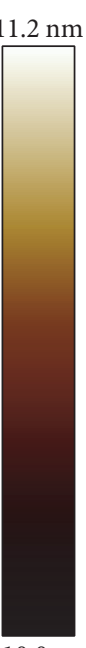

$-10.0 \mathrm{~nm}$

(c)

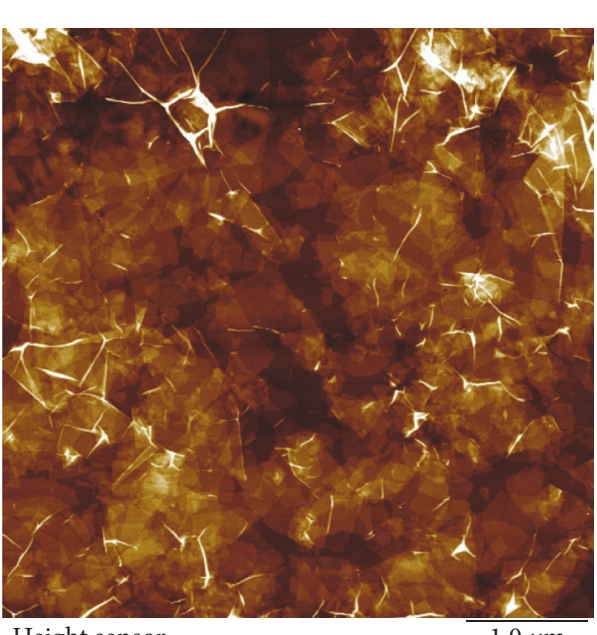

Height sensor

(b)

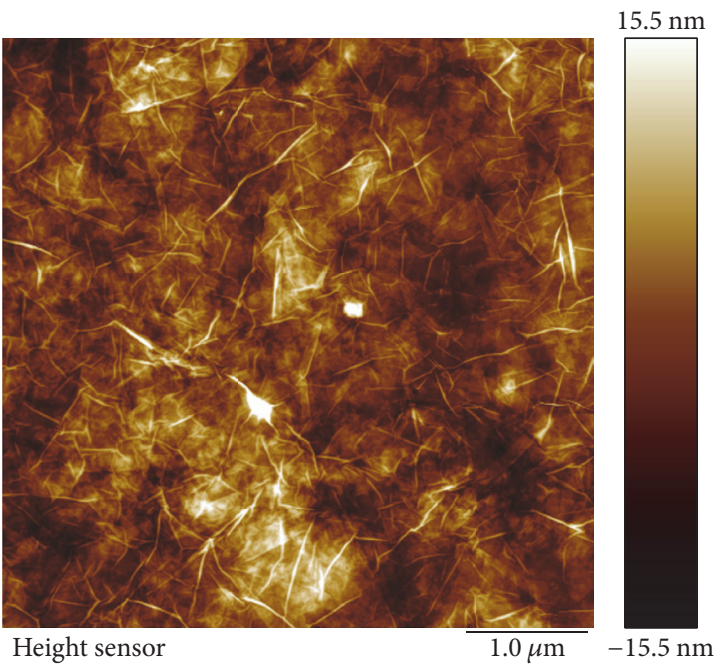

(d)

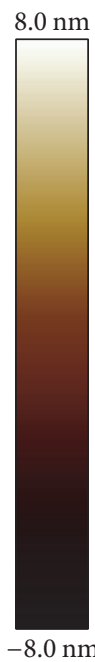

$5 \mathrm{~nm}$

Figure 2: Atomic force microscopy (AFM) GO layers: (a) GO, (b) UV reduction, (c) chemical reduction by hydrazine, and (d) thermal reduction.

in terms of complex refractive index and extinction coefficient are shown in Figure 5. The deviations of ellipsometric measurement are assumed to be due to some roughness effects of the layers and to some variations in thicknesses, so it was necessary to fit the following parameters: $E_{\mathrm{g}}$ is the optical band gap energy, $A$ the strength of the absorption peak, $C$ the broadening term of the peak, and $E$ the energy of maximum transition. The value of $E_{\mathrm{g}}$ was inserted from UV-VIS spectra [41]. The roughness of the substrates used is less than $1 \AA$ (declared by the producer Heraeus), compared with profilometric measurement of GO samples where the roughness is $5 \mathrm{~nm}$. Ellipsometric measurements taken at 6 different locations give an average of $(15 \pm 4) \mathrm{nm}$. The same values were obtained by another method (see AFM measurements) which shows an initial surface roughness of about $3 \mathrm{~nm}$. The summarized data on thicknesses is shown in Table 1.
The fitted model was verified in tests of other films that were similar to the layers investigated by Shen et al. [42]. The fitted parameters are in a good agreement with the data in the literature [42]. The mean squared error (MSE) quantifies the difference between model and experiment for all regressed quantities. The simulated data was fitted to the experimental ellipsometric results with the mean square error value MSE $\sim 0.03$. Summarized data on MSE is shown in the Supplementary Table S1 in Supplementary Material available online at https://doi.org/10.1155/2017/3501903.

The mean values of refractive indexes and extinction coefficients are listed in Table 2. These determined values of all layers obtained by fitting the ellipsometric spectra are in a good correlation with the results obtained by using a mechanical profilometer and AFM (the thicknesses of GO and rGO layers were about $15 \mathrm{~nm}$ for all samples, as shown in Table 1). 


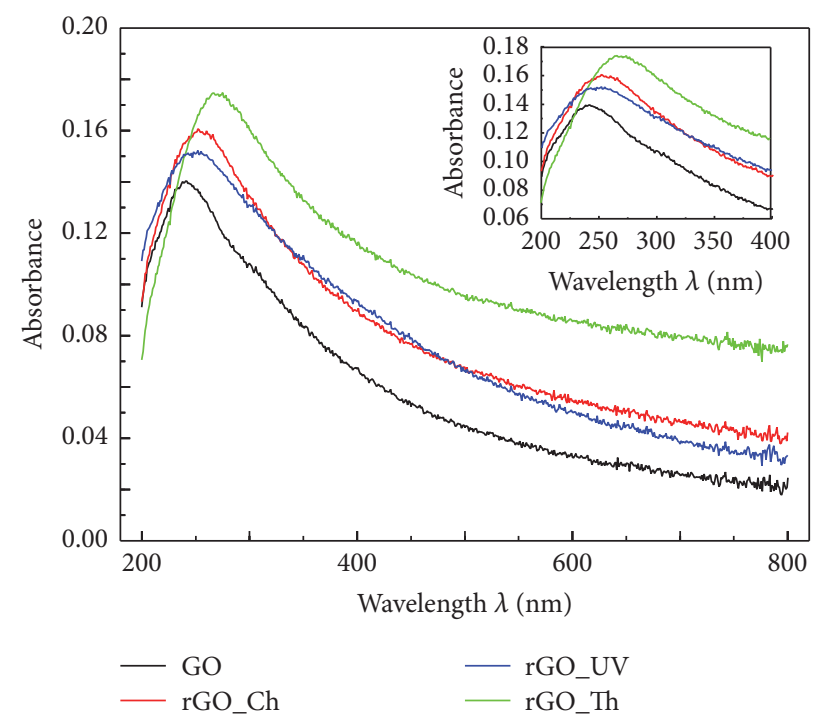

FIGURE 3: Absorption spectra of prepared GO layers (without reduction, black line; with chemical reduction, red line; with UV reduction, blue line; and with thermal reduction, green line).

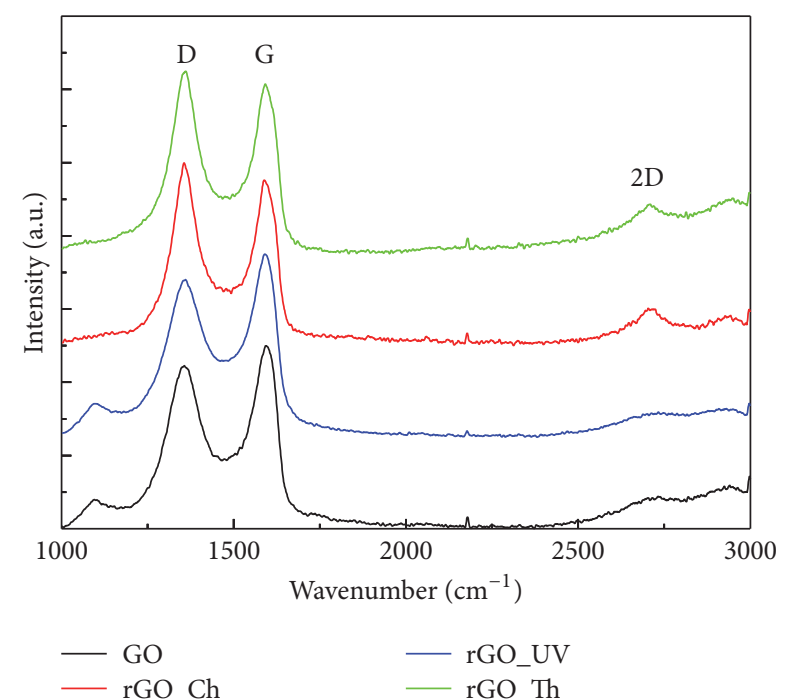

FIGURE 4: Raman spectra of GO layers (without reduction, black line; with chemical reduction, red line; with UV reduction, blue line; and with thermal reduction, green line).

Figure 6 and Supplementary Figure S1 summarize all determined refractive indexes and extinction coefficients and also depict the spectra of the studied GO and its reduction, obtained by spectroscopic ellipsometry.

Optical properties of materials are significant primarily for evaluation of experiments focused on the study of electronic processes.

The measurements of sheet resistance (four-point probe method) were in a good agreement with the optical measurements: sheet resistance decreased with increasing quality of graphene in the case of chemical and thermal reduction (see Table 3). The obtained values correspond to the previously

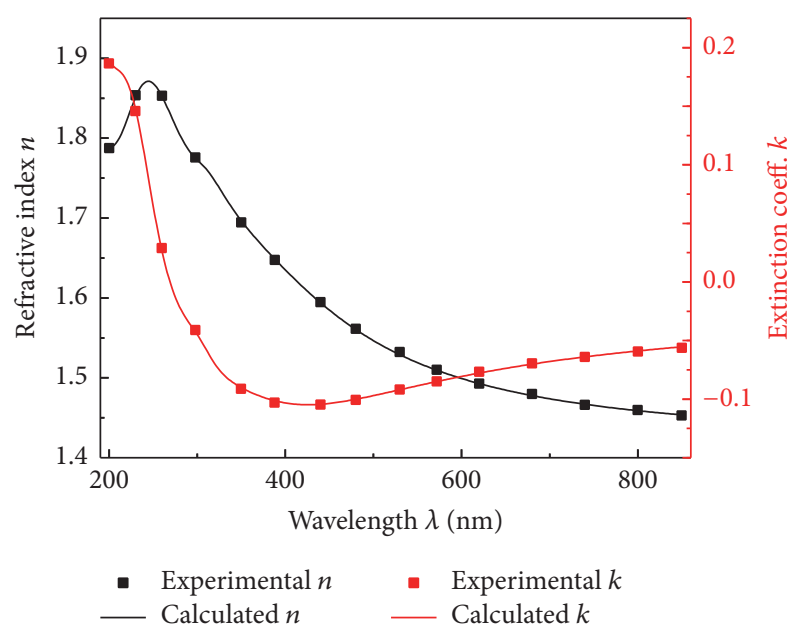

FIgURE 5: Experimental (squares) and calculated (full line) optical spectra of a thin film of GO from the glass side of substrates.

reported ones [21,43]. In the case of chemical reduction, sheet resistance was an order of magnitude higher than in other above-mentioned cases. This may be a result of macroscopic cracks or wrinkles in the rGO layers; thus a charge transfer was hindered in the lateral direction.

Also preparation of organic solar cells was tried with bulk heterojunction based on a 3,6-bis[5-(benzofuran-2-yl)thiophen-2-yl]-2,5-bis(2-ethylhexyl)pyrrolo[3,4-c]pyrrole-1,4-dione and [6,6]-phenyl $\mathrm{C}_{61}$ butyric acid methyl ester $\left(\mathrm{DPP}(\mathrm{TBFu})_{2}: \mathrm{PC}_{60} \mathrm{BM}\right)$ blend with the GO hole-extraction layer reduced in the above-mentioned way. The final structure of these devices can be seen in Supplementary Figure S2. The best measured photovoltaic parameters of such samples are summarized in Supplementary Table S2 and measured $I-V$ characteristic was shown in Figure S2.2. It was found that using a thin $(5 \mathrm{~nm}) \mathrm{GO}$ extraction layer for transporting holes leads to a decrease in power conversion efficiency values in comparison with a reference cell (in the case where PEDOT:PSS is used as a hole transport layer). Similar results have also been published in [44].

\section{Conclusion}

In the present paper a complex characterization of inkjetprinted graphene oxide and reduced graphene oxide thin films using ellipsometry measurements was reported. It was found that thermal reduction is probably the best way of reducing GO while UV reduction and chemical (by hydrazine) reduction result in more defects in the $\mathrm{GO} / \mathrm{rGO}$ structure (the most disordered is chemically reduced GO because, as declared in [30], the hydrazine-assisted chemical reduction results in the decoration of the graphitic lattice with nitrogen-based functionalities), which was confirmed by Raman measurements. AFM measurements on thin film GO and rGO have shown no pinholes or cracks regardless of the reduction method. Roughness was in the range of $2-3 \mathrm{~nm}$ for nonreduced and also reduced samples, but the roughness strongly depends on the thickness of the samples (on the 


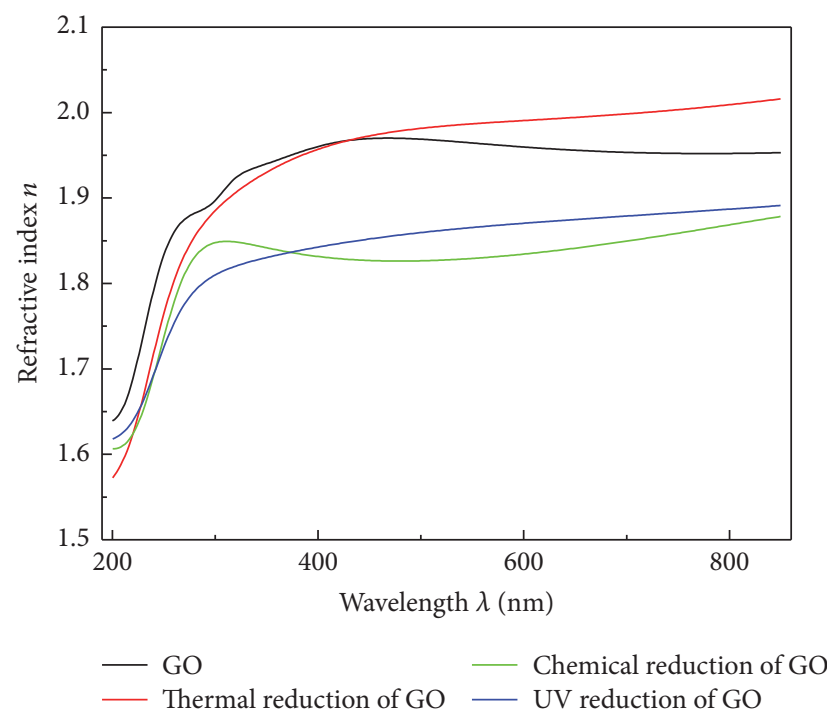

(a)

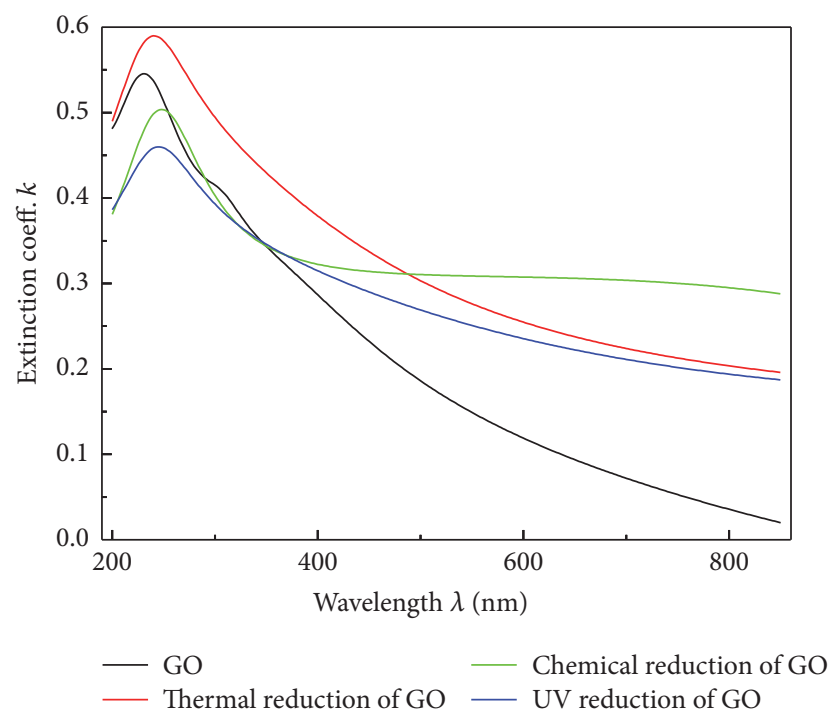

(b)

FIGURE 6: Dependencies of refractive indexes (a) and extinction coefficients (b) on the wavelength of thin films of GO and rGO.

TABLE 1: Thicknesses of the prepared samples determined by spectroscopic ellipsometry (SE), profilometry, and atomic force microscopy (AFM).

\begin{tabular}{lccc}
\hline Material & Thickness SE $[\mathrm{nm}]$ & Thickness profilometer [nm] & Thickness AFM [nm] \\
\hline Graphene oxide & $11.3 \pm 0.6$ & $16.1 \pm 2.5$ & $18.1 \pm 2.3$ \\
Chemical reduction of GO & $19.3 \pm 0.5$ & $18.4 \pm 4.0$ & $19.5 \pm 3.9$ \\
Thermal reduction of GO & $17.7 \pm 0.4$ & $16.8 \pm 5.1$ & $21.5 \pm 5.5$ \\
UV reduction of GO & $13.6 \pm 0.9$ & $15.3 \pm 3.7$ & $16.7 \pm 2.0$ \\
\hline
\end{tabular}

TABLE 2: Optical parameters $(n, k)$ at a $634 \mathrm{~nm}$ wavelength of the prepared samples determined by spectroscopic ellipsometry.

\begin{tabular}{lcc}
\hline Material & Refractive index $n$ & Extinction coeff. $k$ \\
\hline Graphene oxide & 1.957 & 0.101 \\
Chemical reduction of GO & 1.839 & 0.307 \\
Thermal reduction of GO & 1.993 & 0.243 \\
UV reduction of GO & 1.873 & 0.226 \\
\hline
\end{tabular}

TABLE 3: Sheet resistance for graphene oxide thin films and their reduced forms.

\begin{tabular}{lc}
\hline Material & Sheet resistance [Ohm/sq.] \\
\hline Graphene oxide & - \\
Chemical reduction of GO & $5.7 \cdot 10^{4}$ \\
Thermal reduction of GO & $6.8 \cdot 10^{3}$ \\
UV reduction of GO & $2.5 \cdot 10^{3}$ \\
\hline
\end{tabular}

number of GO layers) and also on the type of GO flakes used. The main additional value of the paper is the determination of complex refractive indexes of nonreduced and reduced (thermally, chemically, and by UV irradiation) GO thin films by spectroscopic ellipsometry. All determined values of refractive indexes and extinction coefficients are summarized in Table 1. It was found that the refractive index of unreduced samples is equal to about 1.96, while the refractive indexes of the reduced form of GO differ slightly (being around 1.84 for chemical reduction, 1.87 for UV, and 1.99 for thermally reduced samples). As regards the increasingly frequent use of graphene and graphene-based materials, such as GO, and also due to the tunability of their properties, it is necessary to investigate all relevant parameters of those materials, especially their optical properties.

\section{Conflicts of Interest}

The authors declare that there are no conflicts of interest regarding the publication of this paper. 


\section{Acknowledgments}

This work was supported by the Czech Science Foundation under Project no. 15-05095S. Research infrastructure was supported by Project MŠMT no. LO1211 addressed to the Materials Research Centre at FCH BUT. The authors are also grateful for funding obtained from SAFMAT LM2015088, LO1409, and CZ.2.16/3.1.00/21568.

\section{References}

[1] M. I. Katsnelson, "Graphene: carbon in two dimensions," Materials Today, vol. 10, no. 1-2, pp. 20-27, 2007.

[2] A. H. Castro Neto, F. Guinea, N. M. R. Peres, K. S. Novoselov, and A. K. Geim, "The electronic properties of graphene," Reviews of Modern Physics, vol. 81, no. 1, pp. 109-162, 2009.

[3] D. A. Abanin, K. S. Novoselov, U. Zeitler, P. A. Lee, A. K. Geim, and L. S. Levitov, "Dissipative quantum hall effect in graphene near the dirac point," Physical Review Letters, vol. 98, no. 19, Article ID 196806, 2007.

[4] D. A. Abanin, P. A. Lee, and L. S. Levitov, "Spin-filtered edge states and quantum hall effect in graphene," Physical Review Letters, vol. 96, no. 17, Article ID 176803, 2006.

[5] W. Choi, I. Lahiri, R. Seelaboyina, and Y. S. Kang, "Synthesis of graphene and its applications: a review," Critical Reviews in Solid State and Materials Sciences, vol. 35, no. 1, pp. 52-71, 2010.

[6] K. S. Novoselov, A. K. Geim, S. V. Morozov et al., "Electric field in atomically thin carbon films," Science, vol. 306, no. 5696, pp. 666-669, 2004.

[7] Y. Zhu, S. Murali, W. Cai et al., "Graphene and graphene oxide: synthesis, properties, and applications," Advanced Materials, vol. 22, no. 35, pp. 3906-3924, 2010.

[8] A. Bablich, S. Kataria, and M. C. Lemme, "Graphene and twodimensional materials for optoelectronic applications," Electronics (Switzerland), vol. 5, no. 1, article no. 13, 2016.

[9] R. R. Nair, P. Blake, A. N. Grigorenko et al., "Fine structure constant defines visual transparency of graphene," Science, vol. 320, no. 5881, p. 1308, 2008.

[10] V. G. Kravets, A. N. Grigorenko, R. R. Nair et al., "Spectroscopic ellipsometry of graphene and an exciton-shifted van Hove peak in absorption," Physical Review B, vol. 81, no. 15, Article ID 155413, 2010.

[11] L. Dai, "Functionalization of graphene for efficient energy conversion and storage," Accounts of Chemical Research, vol. 46, no. 1, pp. 31-42, 2013.

[12] S. Park and R. S. Ruoff, "Chemical methods for the production of graphenes," Nature nanotechnology, vol. 4, no. 4, pp. 217-224, 2009.

[13] J. Yang, Y. Zhou, L. Sun, N. Zhao, C. Zang, and X. Cheng, "Synthesis, characterization and optical property of graphene oxide films," Applied Surface Science, vol. 258, no. 12, pp. 5056-5060, 2012.

[14] S. R. Forrest, "The path to ubiquitous and low-cost organic electronic appliances on plastic," Nature, vol. 428, no. 6986, pp. 911-918, 2004.

[15] J. M. Xu, "Plastic electronics and future trends in microelectronics," Synthetic Metals, vol. 115, no. 1, pp. 1-3, 2000.

[16] D. R. Dreyer, S. Park, C. W. Bielawski, and R. S. Ruoff, "The chemistry of graphene oxide," Chemical Society Reviews, vol. 39, no. 1, pp. 228-240, 2010.
[17] K. J. Tielrooij, J. C. W. Song, S. A. Jensen et al., "Photoexcitation cascade and multiple hot-carrier generation in graphene," Nature Physics, vol. 9, no. 4, pp. 248-252, 2013.

[18] G. Jo, M. Choe, S. Lee, W. Park, Y. H. Kahng, and T. Lee, “The application of graphene as electrodes in electrical and optical devices," Nanotechnology, vol. 23, no. 11, Article ID 112001, 2012.

[19] G. Kopidakis, I. N. Remediakis, M. G. Fyta, and P. C. Kelires, "Atomic and electronic structure of crystalline-amorphous carbon interfaces," Diamond and Related Materials, vol. 16, no. 10, pp. 1875-1881, 2007.

[20] Q. Zheng, Z. Li, J. Yang, and J.-K. Kim, "Graphene oxide-based transparent conductive films," Progress in Materials Science, vol. 64, pp. 200-247, 2014.

[21] H. A. Becerril, J. Mao, Z. Liu, R. M. Stoltenberg, Z. Bao, and Y. Chen, "Evaluation of solution-processed reduced graphene oxide films as transparent conductors," ACS Nano, vol. 2, no. 3, pp. 463-470, 2008.

[22] R. K. Singh, R. Kumar, and D. P. Singh, "Graphene oxide: strategies for synthesis, reduction and frontier applications," RSC Advances, vol. 6, no. 69, pp. 64993-65011, 2016.

[23] A. C. Faucett, J. N. Flournoy, J. S. Mehta, and J. M. Mativetsky, "Evolution, structure, and electrical performance of voltagereduced graphene oxide," FlatChem, vol. 1, pp. 42-51, 2017.

[24] S. F. Pei and H.-M. Cheng, "The reduction of graphene oxide," Carbon, vol. 50, no. 9, pp. 3210-3228, 2012.

[25] M. J. Fernández-Merino, L. Guardia, J. I. Paredes et al., "Vitamin $\mathrm{C}$ is an ideal substitute for hydrazine in the reduction of graphene oxide suspensions," Journal of Physical Chemistry C, vol. 114, no. 14, pp. 6426-6432, 2010.

[26] A. Giuri, S. Rella, C. Malitesta et al., "Synthesis of reduced graphite oxide by a novel green process based on UV light irradiation," Science of Advanced Materials, vol. 7, no. 11, pp. 2445-2451, 2015.

[27] X. Wan, G. Long, L. Huang, and Y. Chen, "Graphene-a promising material for organic photovoltaic cells," Advanced Materials, vol. 23, no. 45, pp. 5342-5358, 2011.

[28] G. Venugopal, K. Krishnamoorthy, R. Mohan, and S.-J. Kim, "An investigation of the electrical transport properties of graphene-oxide thin films," Materials Chemistry and Physics, vol. 132, no. 1, pp. 29-33, 2012.

[29] Q. V. Lea, J.-Y. Choib, and S. Y. Kim, "Recent advances in the application of two-dimensional materials as charge transport layers in organic and perovskite solar cells," FlatChem, vol. 2, pp. 54-66, 2017.

[30] L. Sygellou, G. Paterakis, C. Galiotis, and D. Tasis, "Work function tuning of reduced graphene oxide thin films," Journal of Physical Chemistry C, vol. 120, no. 1, pp. 281-290, 2016.

[31] R. Søndergaard, M. Hösel, D. Angmo, T. T. Larsen-Olsen, and F. C. Krebs, "Roll-to-roll fabrication of polymer solar cells," Materials Today, vol. 15, no. 1-2, pp. 36-49, 2012.

[32] J. T. Han, J. S. Kim, D. Kwak et al., "Transparent carbon nanotube patterns templated by inkjet-printed graphene oxide nanosheets," RSC Advances, vol. 1, no. 1, pp. 44-47, 2011.

[33] A. R. Forouhi and I. Bloomer, "Optical dispersion relations for amorphous semiconductors and amorphous dielectrics," Physical Review B, vol. 34, no. 10, pp. 7018-7026, 1986.

[34] A. R. Forouhi and I. Bloomer, "Optical properties of crystalline semiconductors and dielectrics," Physical Review B, vol. 38, no. 3, pp. 1865-1874, 1988.

[35] G. E. Jellison Jr. and F. A. Modine, "Parameterization of the optical functions of amorphous materials in the interband region," Applied Physics Letters, vol. 69, no. 3, pp. 371-373, 1996. 
[36] J. Shang, L. Ma, J. Li, W. Ai, T. Yu, and G. G. Gurzadyan, “The origin of fluorescence from graphene oxide," Scientific Reports, vol. 2, article no. 792, 2012.

[37] V. Schmiedova, J. Pospisil, O. Zmeskal, and V. Vretenar, “Optical characterization of graphene oxide films by spectroscopic ellipsometry," Materials Science Forum, vol. 851, pp. 199-204, 2016.

[38] Q. Mei, J. Chen, J. Zhao et al., "Atomic Oxygen Tailored Graphene Oxide Nanosheets Emissions for Multicolor Cellular Imaging," ACS Applied Materials and Interfaces, vol. 8, no. 11, pp. 7390-7395, 2016.

[39] Y. Wang, D. C. Alsmeyer, and R. L. McCreery, "Raman spectroscopy of carbon materials: Structural basis of observed spectra," Chemistry of Materials, vol. 2, no. 5, pp. 557-563, 1990.

[40] L. M. Malard, M. A. Pimenta, G. Dresselhaus, and M. S. Dresselhaus, "Raman spectroscopy in graphene," Physics Reports, vol. 473, pp. 51-87, 2009.

[41] V. Schmiedova, P. Heinrichova, O. Zmeskal, and M. Weiter, "Characterization of polymeric thin films for photovoltaic applications by spectroscopic ellipsometry," Applied Surface Science, vol. 349, pp. 582-588, 2015.

[42] Y. Shen, P. Zhou, Q. Q. Sun et al., "Optical investigation of reduced graphene oxide by spectroscopic ellipsometry and the band-gap tuning," Applied Physics Letters, vol. 99, no. 14, Article ID 141911, 2011.

[43] J. B. Wu, H. A. Becerril, Z. N. Bao, Z. F. Liu, Y. S. Chen, and P. Peumans, "Organic solar cells with solution-processed graphene transparent electrodes," Applied Physics Letters, vol. 92, no. 26, pp. 263302-263303, 2008.

[44] X. Liu, H. Kim, and L. J. Guo, "Optimization of thermally reduced graphene oxide for an efficient hole transport layer in polymer solar cells," Organic Electronics: physics, materials, applications, vol. 14, no. 2, pp. 591-598, 2013. 

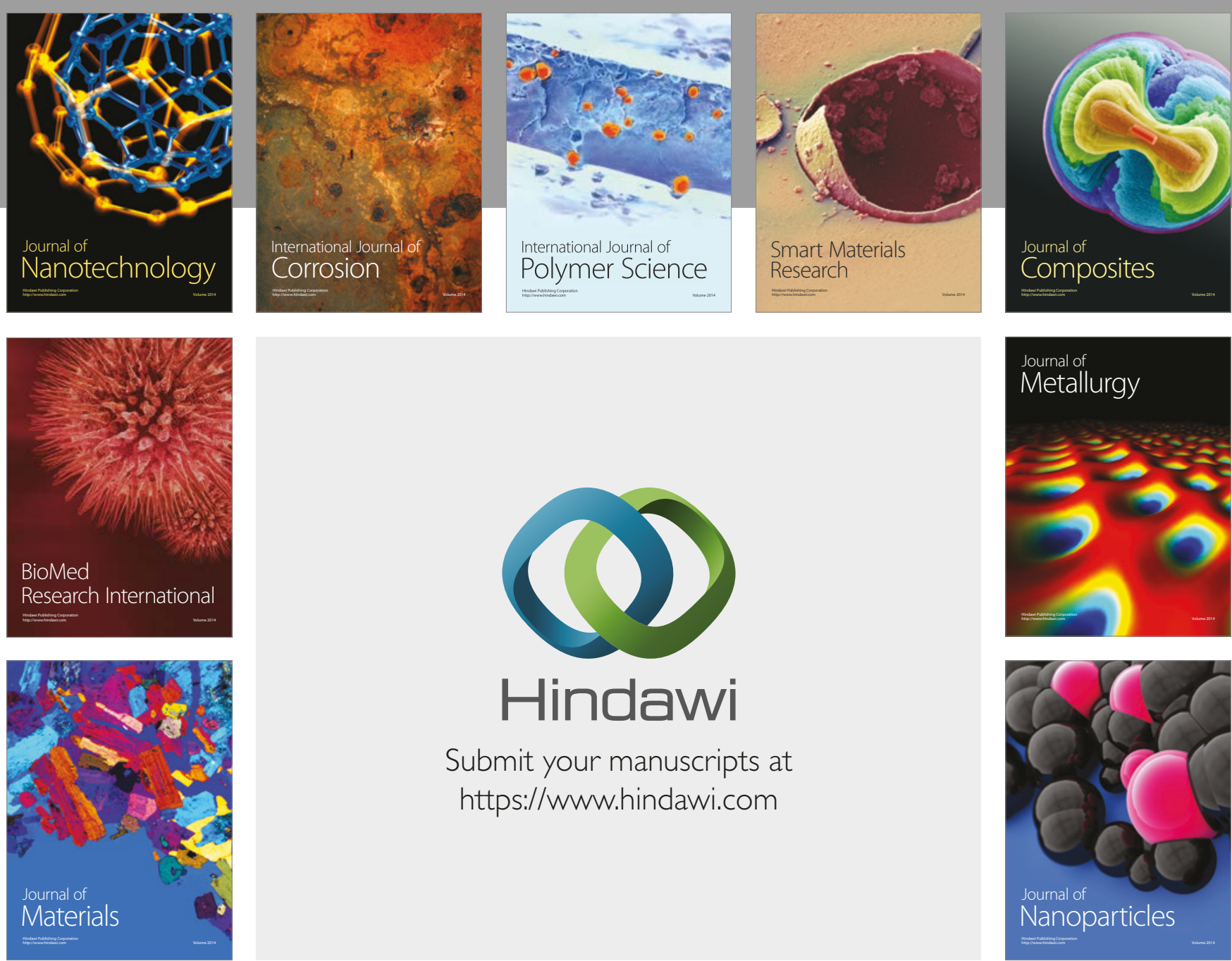

\section{Hindawi}

Submit your manuscripts at

https://www.hindawi.com
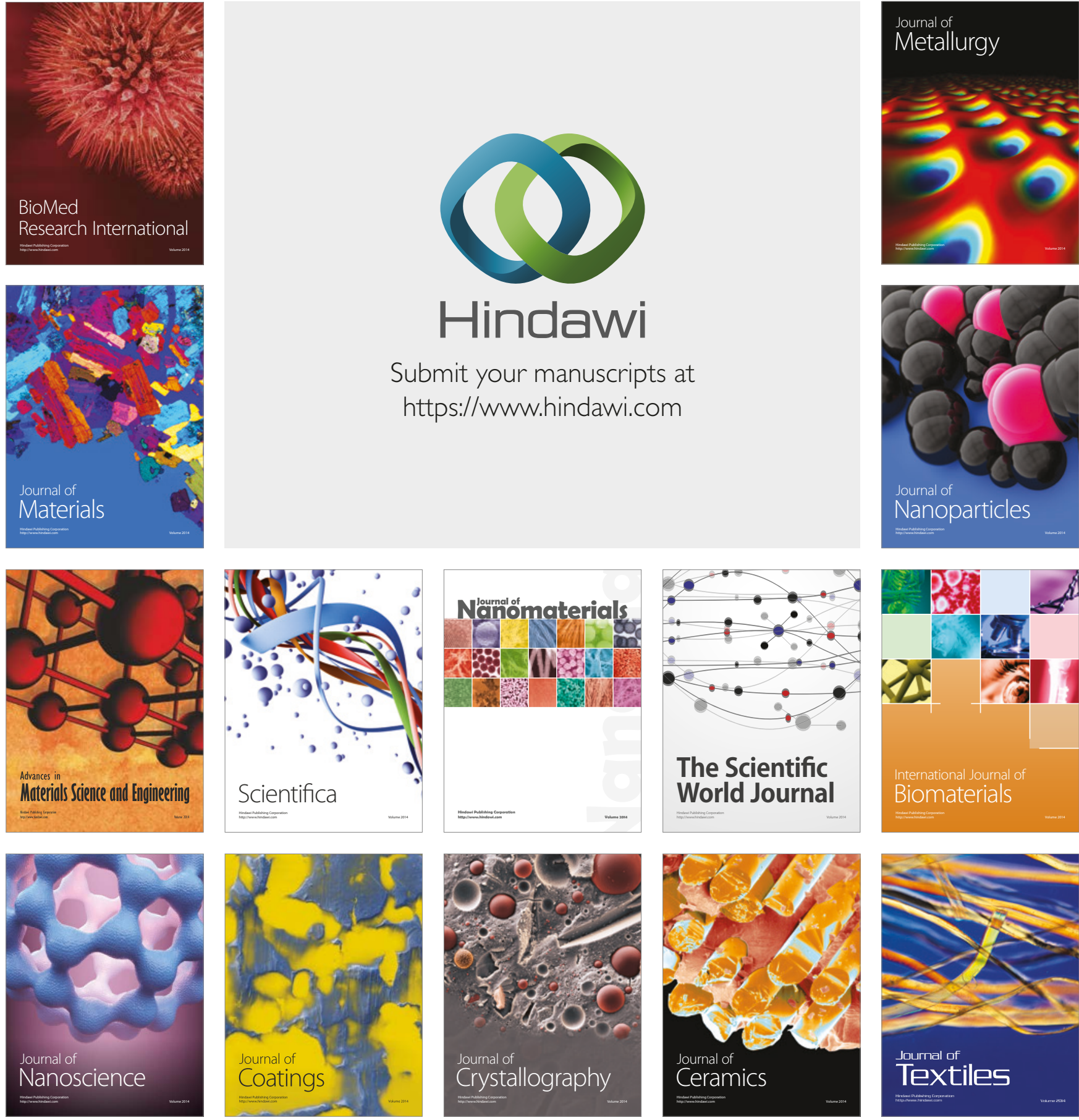

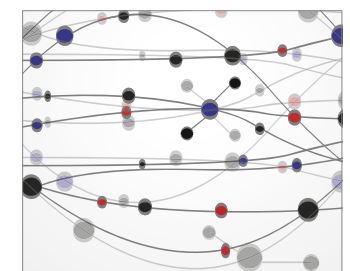

The Scientific World Journal
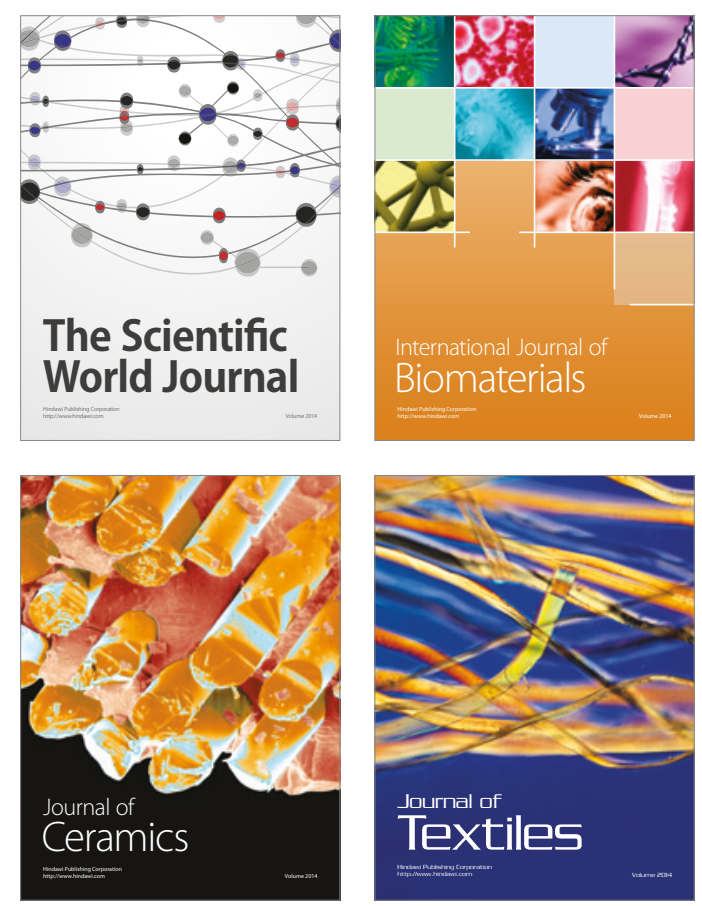